\title{
Epigenetic analysis of FHL1 tumor suppressor gene in human liver cancer
}

\author{
JUN WANG* ${ }^{*}$ FANG HUANG ${ }^{*}$, JIAN HUANG, JINDAN KONG, SHENGLAN LIU and JUN JIN \\ Department of Intensive Care Unit, The First Affiliated Hospital of Soochow University, Suzhou, Jiangsu 215006, P.R. China
}

Received August 16, 2015; Accepted July 27, 2017

DOI: $10.3892 / 01.2017 .6950$

\begin{abstract}
Liver cancer is one of the most common types of cancer among human malignancies. Four and a half LIM domains 1 (FHL1), as a tumor suppressor gene, is frequently downregulated in multiple types of human cancer. However, the role and specific mechanisms of FHL1 as a tumor suppressor in liver cancer are poorly understood. The present study aimed to investigate the role and associated mechanisms of FHL1 in human liver cancer. The level of FHL1 mRNA in hepatocellular carcinoma (HCC) tissue specimens and cell lines derived from the human liver was determined using reverse transcription polymerase chain reaction and western blot analysis. The association between FHL1 expression and clinicopathological characteristics of patients with liver cancer was analyzed. Western blotting, small interfering RNA (siRNA) and chromatin immunoprecipitation were used to study the expression association of FHL1 and enhancer of zeste homolog 2 (EZH2) in human liver cancer and to explore the regulatory mechanism of FHL1 downregulation. Colony formation and migration assays were performed while FHL1 was overexpressed in Hep3B cells. The results showed that the expression of FHL1 mRNA in tumor tissue decreased, exhibiting a significant difference compared with the adjacent non-cancerous tissue $(\mathrm{P}<0.05)$. However, the downregulation of FHL1 was not significantly associated with the sex, age, hepatitis B virus infection status, tumor size, distant metastasis status or level of tumor differentiation of the patients. FHL1 was synergistically silenced by DNA methylation and histone modification, and 3-deanzaneplanocin A (DZNep), an inhibitor of EZH2, which is a histone methyltransferase of the polycomb repressive complex 2, which catalyzes histone H3 lysine 27 tri-methylation (H3K27me3). A significant
\end{abstract}

Correspondence to: Dr Jun Jin, Department of Intensive Care Unit, The First Affiliated Hospital of Soochow University, 188 Shizi Street, Suzhou, Jiangsu 215006, P.R. China

E-mail: jinjundoctor@163.com

*Contributed equally

Key words: FHL1 tumor-suppressor gene, methylation, EZH2, H3K $27 \mathrm{me} 3$ association between FHL1 and EZH2 expression was identified in the female hepatocellular carcinoma (HCC) samples, but was not in the male HCC samples. FHL1 overexpression and DZNep treatment significantly suppressed the growth and migration of Hep3B cells by restoring FHL1 expression. H3K27me3 was significantly enriched at the FHL1 promoter region, as indicated by a chromatin immunoprecipitation assay, and associated with the epigenetic repression of the FHL1 tumor suppressor gene in HCC cell lines. In conclusion, the present study provides an insight into DNA methylation and EZH2-H3K27me3 epigenetic repression of FHL1 in human liver cancer.

\section{Introduction}

Primary liver cancer is one of the most frequently occurring malignancies worldwide, and therefore a major public health challenge (1). The development of liver cancer is a complex, multistep process that is derived from a series of genetic and epigenetic alternations. The inactivation of tumor suppressor genes as a result of aberrant DNA methylation and histone modification is a characteristic step in tumor development and progression (2). Furthermore, the downregulation of the epigenetic regulator led to epigenetic alternations and contributed to abnormal inactivation of tumor suppressor genes in numerous types of human cancer $(3,4)$. Accordingly, improved understanding the molecular basis of liver cancer may enhance the development of novel strategies to improve the treatment of liver cancer.

The four and a half LIM domains (FHL) family has an important role in regulating cell proliferation, differentiation and apoptosis, and the member FHL1, located on human chromosome Xq26, functions in skeletal and cardiac muscle growth $(5,6)$. Numerous studies of clinical samples have shown that FHL1 expression was downregulated in multiple types of malignancy, including lung (7), gastric $(8,9)$, breast, kidney and prostate cancers (10). Notably, FHL1 as tumor suppressor gene on chromosome $\mathrm{X}$ has a high risk to be affected as a single hit of genetic and/or epigenetic abnormality on only one active allele could lead to complete inactivation of FHL1 (11). FHL1 exerts a tumor suppressor effect via multiple mechanisms, including the activation of the transforming growth factor- $\beta$-like and mitogen-activated protein kinase signaling pathways and protein interaction with zonula occludens-1, hypoxia-inducible factor $1-\alpha$ and estrogen receptor $\alpha(7,9,12,13)$. However, the 
role of FHL1 in liver cancer has not been revealed and the mechanisms associated with FHL1 downregulation remain unknown.

Enhancer of zeste homolog 2 (EZH2) is a frequently elevated epigenetic regulator as the catalytic subunit of polycomb repressive complex 2 (PRC2) in multiple types of human cancer (14-16). As a histone methyltransferase, EZH2 specifically catalyzes histone $\mathrm{H} 3$ lysine 27 tri-methylation (H3K27me3), a repressive histone modification, to epigenetically control gene transcription (17). EZH2 serves an oncogenic role in different types of human cancer, primarily through the epigenetic silencing of tumor suppressor genes: For example, EZH2-mediated trimethylation of histone H3 at lysine 27 (H3K27me3) epigenetically silenced chromodomain helicase DNA binding protein 5, which serves as a tumor suppressor in HCC cells (18). Overexpression of EZH2 may lead to hypermethylation of p16 INK4a promoter, followed by a decreased expression of p16 INK4a in the multi-step cholangiocarcinogenesis (19). To address the mechanism of FHL1 downregulation during HCC genesis, the present study investigated the epigenetic dysregulation and related effects of FHL1. The data demonstrated that FHL1 was synergistically silenced by DNA methylation and histone modification, and revealed the epigenetically regulatory mechanisms by which FHL1 was inactivated during hepatocarcinogenesis.

\section{Materials and methods}

Cell lines and tissue specimens. The human HCC-derived MHCC-97L and Hep3B, human liver LO-2 and HepG2, which was re-identified as a human hepatoblastoma cell line (20), cell lines were obtained from the Cell Bank of Chinese Academy of Sciences (Shanghai, China). All cells were cultured routinely in Dulbecco's modified Eagle's medium (DMEM) supplemented with heat-inactivated fetal bovine serum (FBS; Gibco; Thermo Fisher Scientific, Inc., Waltham, MA, USA), penicillin $(100 \mathrm{U} / \mathrm{ml})$ and streptomycin $(100 \mu \mathrm{g} / \mathrm{ml})$ at $37^{\circ} \mathrm{C}$ in a $5 \% \mathrm{CO}_{2}$-humidified chamber for $24 \mathrm{~h}$. The tissue samples used in the present study were obtained from the First Affiliated Hospital of Soochow University (Suzhou, China). All experimental protocols were approved by Soochow University Ethics Committee (Suzhou, China) and all patients provided written informed consent.

Reverse transcription quantitative polymerase chain reaction (RT-qPCR). Total RNA was extracted from the Hep3B, HepG2 cells and liver cancer tissues using TRIzol reagent (Thermo Fisher Scientific, Inc.) and reverse transcribed using a Superscript III kit (Thermo Fisher Scientific, Inc.). RT-qPCR was performed using an SYBR-Green qPCR Master mix (Thermo Fisher Scientific, Inc.). An initial denaturation was performed for $5 \mathrm{~min}$ at $94^{\circ} \mathrm{C}$, and 35 cycles were performed with the following PCR program: Denaturation at $94^{\circ} \mathrm{C}$ for $30 \mathrm{sec}$, annealing at $55^{\circ} \mathrm{C}$ for $30 \mathrm{sec}$ for $\mathrm{FHL} 1$ and $55^{\circ} \mathrm{C}$ for $30 \mathrm{sec}$ for $\beta$-actin and elongation at $72^{\circ} \mathrm{C}$ for $30 \mathrm{sec}$. The upstream and downstream FHL1 primers were 5'-ACAATC CTGGCACGACTA-3' and 5'-AAAATGGGAGAAAAG ACG-3', respectively. Housekeeping gene $\beta$-actin was used as reference gene, its upstream and downstream primers were 5'-TCACCAACTGGGACGACA-3' and 5'-TGCAAAGAA
CACGGCTAA-3'. Gene expression levels were normalized to $\beta$-actin, and the fold change of target genes was calculated using $2^{-\Delta \Delta \mathrm{Cq}}(21)$.

Western blotting. Total proteins were extracted using radioimmunoprecipitation lysis buffer [150 mM NaCl, $1 \%$ NP40, $0.5 \%$ sodium deoxycholate, $0.1 \%$ SDS, $50 \mathrm{mM}$ Tris (pH 7.9), $10 \mathrm{mM}$ NaF, PMSF and protease inhibitors (Complete Cocktail tablets, Roche Diagnostics, Basel, Switzerland)]. Protein concentrations were measured using Pierce ${ }^{\mathrm{TM}}$ BCA Protein Assay kit (Thermo Fisher Scientific, Inc.). These extracts $(50 \mu \mathrm{g})$ were subjected to electrophoresis by $10 \%$ SDS-PAGE and then transferred onto Hybrid-P polyvinylidene difluoride membrane (Merck KGaA, Darmstadt, Germany). Following blocking with PBS containing 5\% skimmed milk powder and $0.1 \%$ Tween-20 for $2 \mathrm{~h}$ at room temperature, the blot was incubated for immunoblotting analysis with antibodies against FHL1 (cat. no. sc-374246; 1:1,000 dilution; Santa Cruz Biotechnology, Inc., Dallas, TX, USA), H3K27me3 (cat. no. 9733S; 1:500 dilution; Cell Signaling Technology, Inc., Danvers, MA, USA) or $\beta$-actin (cat. no. sc-47778; 1:2,000 dilution; Santa Cruz Biotechnology, Inc.,) overnight at $4^{\circ} \mathrm{C}$. Peroxidase-conjugated secondary antibodies (cat. nos. SAB3701171 and SAB3700831; 1:5,000 dilution; Sigma-Aldrich; Merck KGaA, Darmstadt, Germany) were used, and membranes were developed using the enhanced chemiluminescent immunoassay (Thermo Fisher Scientific, Inc.) for the detection of antigens.

Adenovirus preparation. The recombinant adenovirus carrying FHL1 (pAd-FLH1) or green fluorescent protein (GFP) was generated using Ad-Easy system according to the manufacturer's protocol (Stratagene; Agilent Technologies, Inc., Santa Clara, CA, USA). For packaging of the adenoviruses expressing FHL1 or GFP, pAd-FHL1 and pAd-GFP was transfected into AD293 cells using Lipofectamine $2000^{\circledR}$ reagent (Thermo Fisher Scientific Inc.). The adenoviruses were amplified in $\mathrm{AD} 293$, purified, titrated and stored at $-80^{\circ} \mathrm{C}$ until use.

Bisulfite sequencing. Genomic DNA was isolated using DNeasy Tissue kit (Qiagen, Inc., Valencia, CA, USA), and bisulfite modification was performed with the EpiTect Bisulfite kit (Qiagen, Inc.) according to the manufacturer's protocol. $\mathrm{CpG}$ enrichment region of the FHL1 promoter was analyzed by Cpgplot (http://www.ebi.ac.uk/Tools/seqstats/emboss_cpgplot/). The PCR products were cloned into PMD 18-T vector (Takara Bio, Inc., Otsu, Japan), and 8 clones were randomly selected for each specimen DNA sequencing.

Chromatin immunoprecipitation (ChIP) assay. A ChIP assay was performed using an EZ ChIP kit (EMD Millipore, Billerica, MA, USA) according to the manufacturer's protocol, with slight modifications. The Hep3B and HepG2 cells were cross-linked in $1 \%$ formaldehyde and quenched with the addition of $125 \mathrm{mM}$ glycine. Subsequent to washing twice in ice-cold PBS containing a protease inhibitor cocktail, the cell lysates were harvested in ChIP lysis buffer [50 mM HEPEs ( $\mathrm{pH}$ 7.5), 1 mM EDTA (pH 8.0), $150 \mathrm{mM}$ sodium chloride, $0.1 \%$ sodium deoxycholate, $0.1 \%$ SDS, $1 \%$ Triton X-100 and complete phenylmethylsulfonyl fluoride]. Subsequently, chromatin was 
sheared to fragments of 300-500 bp by sonication 9 times for 10-20 sec at $80 \%$ setting using VibraCell Sonicator (Sonics \& Materials, Newtown, CT, USA) at a frequency of $20 \mathrm{kHz}$. The lysates were pre-cleared with Salmon Sperm DNA/Protein G Agarose (Roche Diagnostics) for $2 \mathrm{~h}$. Samples were centrifuged at $12,000 \mathrm{x}$ g for $15 \mathrm{~min}$ at $4^{\circ} \mathrm{C}$, and then the supernatant was used for immunoprecipitation at $4^{\circ} \mathrm{C}$ overnight with $5 \mu \mathrm{g}$ anti-H3K27me3 (cat. no. 9733S; 1:50 dilution) or anti-mouse IgG (cat. no. 5415; 1:50 dilution; both from Cell Signaling Technology, Inc.) as a negative control. Immunoprecipitated crosslinking DNA fragments were reversed by pronase and subsequently incubated at $42^{\circ} \mathrm{C}$ for $2 \mathrm{~h}$ and $68^{\circ} \mathrm{C}$ for $8 \mathrm{~h}$. The FHL1 promoter DNA in the immunoprecipitates was detected by qPCR using the following primers: Forward, 5'-ACCGAG TGAGAAAAGCCAAT-3' and reverse, 5'-TCACCATTG GCAACCACTGAT-3'. The FHL1 signals were normalized to GAPDH (forward, 5'-TACTAGCGGTTTTACGGGCG-3' and reverse, 5'-TCGAACAGGAGGAGCAGAGAGCGA-3') using the $2^{-\triangle \Delta C q}$ method (21) to determine whether the immunoprecipitate was enriched. qPCR protocol and reactions were performed according to the protocol of the EZ-ChIP Kit (EMD Millipore). Specifically, $25 \mu 1$ PCR reaction system including $2 \mu \mathrm{l}$ of immunoprecipitate samples, $12.5 \mu \mathrm{l} \mathrm{SYBR-Green}$

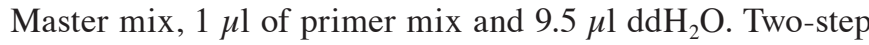
qPCR parameters were set as follows: Initial denaturation at $94^{\circ} \mathrm{C}$ for $10 \mathrm{~min}$, then denaturation at $94^{\circ} \mathrm{C}$ for $20 \mathrm{sec}$, then two-step annealing/extension for $1 \mathrm{~min}$ at $60^{\circ} \mathrm{C}$.

In vitro epigenetic drug treatment. The 3-deanzaneplanocin A (DZNep; Cayman Chemical Company, Ann Arbor, MI, USA) was dissolved in dimethyl sulfoxide (DMSO) and 5-Aza-2' deoxycytidine (5-Aza-dC; Sigma-Aldrich; Merck $\mathrm{KGaA}$ ) was dissolved in 50\% acetic acid. Trichostatin A (TSA; Sigma-Aldrich; Merck KGaA) was dissolved in ethanol. The solvents (DMSO, acetic acid, and ethanol) were used as controls in the corresponding treatment. For the DZNep treatment, DZNep $(10 \mu \mathrm{M})$ was added to the culture medium for 48 or $72 \mathrm{~h}$. For the 5-Aza-dC treatment, 5-Aza-dC $(10 \mu \mathrm{M})$ was replenished daily for $72 \mathrm{~h}$. For the TSA treatment, TSA $(0.25 \mu \mathrm{g} / \mathrm{ml})$ was only added to the cells in the last $24 \mathrm{~h}$ of the experiment.

Colony formation assay. A total of $5 \times 10^{4}$ Hep3B cells were cultured in DMEM media supplemented with $10 \%$ heat-inactivated fetal bovine serum (FBS; Gibco; Thermo Fisher Scientific, Inc.), penicillin (100 U/ml) and streptomycin $(100 \mu \mathrm{g} / \mathrm{ml})$ at $37^{\circ} \mathrm{C}$ in a $5 \% \mathrm{CO}_{2}$-humidified chamber in $10 \mathrm{~cm}$ dishes in triplicate. A total of 3 weeks later, the anchorage-dependent colonies were washed twice with PBS and stained with crystal violet $(0.5 \% \mathrm{w} / \mathrm{v})$.

Transwell migration assay. In total, $1 \times 10^{5} \mathrm{Hep} 3 \mathrm{~B}$ cells were seeded in triplicate Boyden chambers with an $8 \mu \mathrm{m}$-pore sized membrane in the top chamber (BD Biosciences, Franklin Lakes, NJ, USA) in serum-free DMEM media (Thermo Fisher Scientific, Inc.). Media containing 10\% FBS (Thermo Fisher Scientific, Inc.) was used in the bottom chambers. Subsequent to $48 \mathrm{~h}$ incubation, the cells in the upper chamber were wiped, and the migrated cells on the lower surface of the membrane were fixed, stained with $0.1 \%$ crystal violet and photographed using a light microscope (magnification, x20).
Statistical analysis. The differences between two groups in gene expression, colony number and migrated cell number were evaluated using Student's t-test. The statistical significance of differences in multiple groups was determined by analysis of variance with Bonferroni test. Data in histograms was shown as the mean \pm standard deviation from 3 independent replicates. The comparison of the methylated $\mathrm{CpG}$ percentage between tumor and non-cancerous specimens was performed by $\chi^{2}$ test. All statistical analyses were conducted using GraphPad Prism software 5.0 (GraphPad Software, Inc., La Jolla, CA, USA). $\mathrm{P}<0.05$ was considered to indicate a statistically significant difference.

\section{Results}

FHL1 expression was frequently downregulated in patients with liver cancer. The present study examined the expression of FHL1 mRNA in a cohort of 49 paired specimens from liver cancer patients RT-qPCR. The data showed that the FHL1 mRNA level significantly decreased in tumor tissue compared with the matched non-cancerous tissues of patients with liver cancer $(\mathrm{P}<0.001$; Fig. 1A). Of the 49 paired liver cancer specimens examined, $42(85.7 \%)$ exhibited at least a 2 -fold downregulation of FHL1 expression compared with that of the matched non-cancerous liver tissue (Table I), and 8 exhibited a marked difference between tumor and matched non-cancerous tissue, as confirmed by qPCR (Fig. 1B). In addition, the present study analyzed the association between FHL1 expression and clinical factors. However, FHL1 downregulation was not significantly associated with the sex, age, hepatitis B virus, tumor size, metastasis and Edmondson-Steiner grading system (22). The results of western blotting showed that FHL1 exhibited less expression in three liver cancer cell lines, consisting of MHCC-97L, Hep3B and HepG2, one of the most popular hepatoblastoma cell lines (20), than the immortal liver LO2 and WRL68 cell lines. These data showed that FHL1 was downregulated in human liver cancer, which is consistent with the observations of previous studies (12).

FHL1 expression was synergistically by DNA methylation and histone modification. Since DNA methylation and histone modifications are closely associated with respect to establishing a less permissive chromatin status to suppress gene transcription, the present study sought to reveal the combined effect of different epigenetic machineries associated with FHL1 downregulation in liver cancer. Two types of liver cancer cell lines were used, consisting of the hepatocellular carcinoma-derived Hep3B cell line and the hepatoblastoma-derived HepG2 cell line, to investigate the epigenetic effects on FHL1. The two cell lines were treated with DZNep, a small molecular EZH2 inhibitor, and 5-Aza-dC and Trichostatin A TSA, well characterized DNA methylation and histone acetylation inhibitors. As expected, the individual or combined treatments significantly led to increased FHL1 expression $(\mathrm{P}<0.05)$. While treatment of DZNep, 5-Aza-dC and TSA individually elevated expression FHL1, combined treatment of the 3 drugs synergistically restored FHL1 expression in Hep3B cells (Fig. 2A). Additionally, only TSA treatment alone did not restore FHL1 expression and the co-treatment did not induce a further increase in FHL1 
A

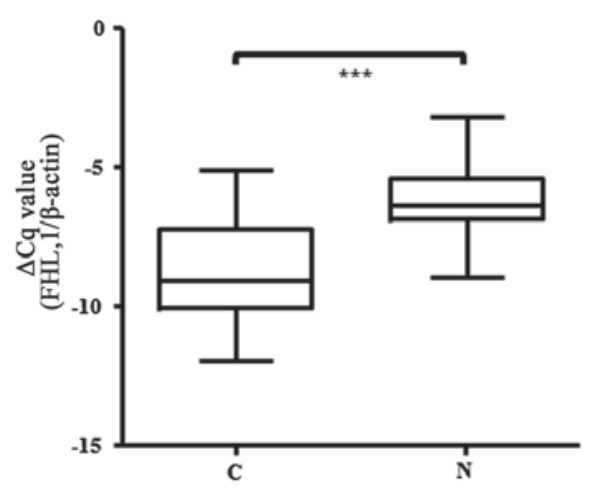

B

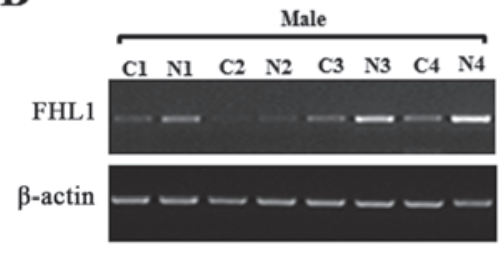

C

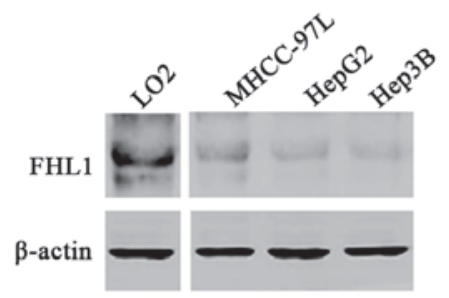

Figure 1. Expression analysis of FHL1 in HCC specimens and cell lines. (A) The transcript level of FHL1 in 49 HCC samples and adjacent non-tumor liver tissues was evaluated by RT-qPCR. The relative mRNA level of FHL1 was normalized based on that of an internal reference $\beta$-actin. The line within each box represents the median- $\Delta \mathrm{Cq}$ value; the upper and lower edges of each box represent the 75 and 25 th percentile, respectively; the upper and lower bars indicate the highest and lowest values, respectively. P-value was calculated by two-tailed Student's t-test. ${ }^{* * *} \mathrm{P}<0.001$. (B) Representative RT-qPCR results of 8 pairs of HCC and corresponding non-tumorous livers. $\beta$-actin was employed as a loading control. (C) Protein expression of FHL1 was evaluated in liver cancer cell lines using western blotting. RT-qPCR, reverse transcription-quantitative polymerase chain reaction; HCC, hepatocellular carcinoma; C, HCC specimens; N, adjacent non-cancerous livers; FLH1, four and a half LIM domains 1.

Table I. Association between clinicopathological characteristics and FHL1 expression in 49 HCC specimens.

\begin{tabular}{lccc}
\hline & \multicolumn{2}{c}{ FHL1 expression } & \\
\cline { 2 - 3 } Characteristics & Decrease & No change & P-value \\
\hline Sex & & & 1.00 \\
Male & 32 & 5 & \\
Female & 10 & 2 & \\
Age & & & 0.45 \\
$<40$ & 6 & 0 & \\
$40-50$ & 11 & 3 & \\
$>50$ & 25 & 4 & \\
HBV & & & 0.34 \\
Positive & 37 & 7 & \\
Negative & 5 & 0 & \\
Tumor size (T) & & & 0.15 \\
T1+T2 & 38 & 6 & \\
T3+T4 & 4 & 1 & \\
Distant metastases (M) & & & 0.47 \\
M0 & 39 & 7 & \\
M1 & 3 & 0 & \\
Edmondson & & & 1.00 \\
I+II & 6 & 1 & \\
III+IV & 36 & 6 & \\
\hline
\end{tabular}

FHL1, four and a half LIM domains 1; HBV, hepatitis B virus.

expression compared with 5-Aza-dC alone in HepG2 cells (Fig. 2B), suggesting that DNA methylation and histone methylation have a crucial epigenetic role in mediating FHL1 downregulation.
EZH2 knockdown restored FHL1 expression. The present study hypothesized that aberrant histone methylation may contribute to FHL1 silencing in HCC cell lines. To investigate whether FHL1 expression could be restored subsequent to the knockdown of EZH2, 2 siRNAs against EZH2 were employed to silence endogenous EZH2 expression. FHL1 was transcriptionally induced in Hep3B ( $\mathrm{P}<0.01$; Fig. 3A) and HepG2 cells ( $\mathrm{P}<0.01$; Fig. 3B), indicating that EZH2-mediated H3K27me3 contributes to the suppression of FHL1 in Hep3B and HepG2 cells. As expected, EZH2 knockdown resulted in a reduction in the level of H3K27me3 (Fig. 3C), and a ChIP assay was performed to assess the enrichment of transcriptional repressive histone modifications H3K27me3 on the FHL1 promoter in Hep3B and HepG2 cells ( $\mathrm{P}<0.01$; Fig. 3D). The findings indicated that the epigenetic silencing of FHL1 by EZH2-mediated $\mathrm{H} 3 \mathrm{~K} 27 \mathrm{me} 3$ is an important mechanism in human liver cancer.

Methylation of FHL1 promoter in liver cancer specimens. To further assess the association between the FHL1 downregulation and the methylation status of the potential methylation positions of the FHL1 gene using $\mathrm{CpG}$ plot arithmetic. As a result, a typical $\mathrm{CpG}$ island (-668 to +234$)$ was found within the promoter and exon of the FHL1 gene. A total of 2 DNA fragments located on the $\mathrm{CpG}$ island were amplified, and bisulfite sequencing was performed to analyze the methylation status in the 8 paired HCC specimens with FHL1 downregulation. The results indicated that the methylation level of the CpG island was significantly enriched in the 4 male HCC specimens compared with the matched non-tumorous liver tissue $(\mathrm{P}<0.001$; Fig. 4). However, no significant difference was identified between tumors and matched non-tumorous specimens in the 4 female patients $(\mathrm{P}=0.604$; Fig. 4). The data propose a hypothesis that other epigenetic mechanisms contribute to FHL1 deregulation in female patients with HCC.

Association between FHL1 and EZH2 expression in human liver cancer tissues. To determine the association between 
$\mathbf{A}$

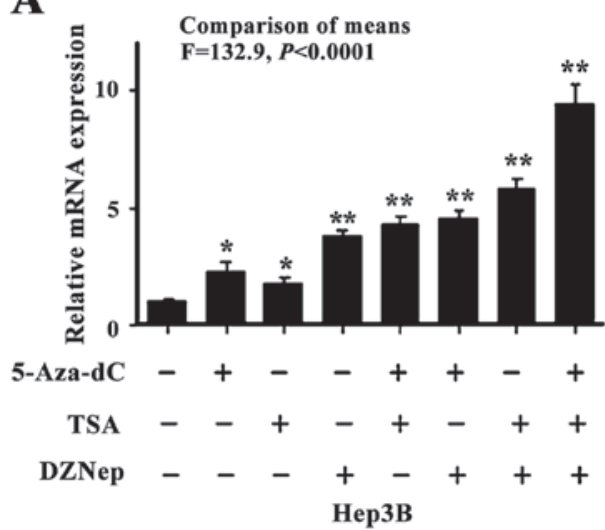

B

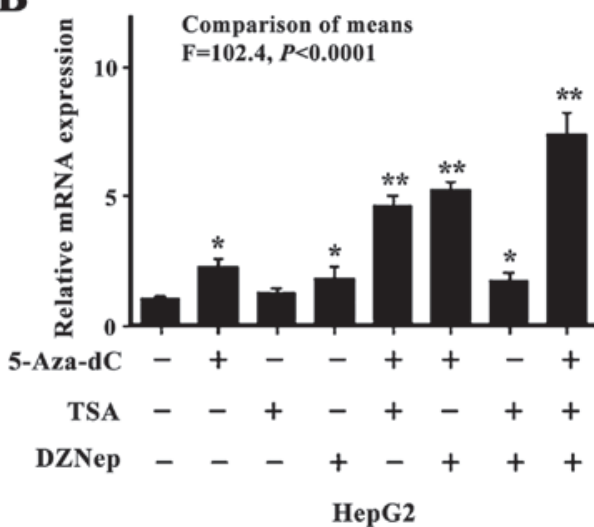

Figure 2. Four and a half LIM domains 1 expression was synergistically restored by DNA methylation and histone modification. (A) Hep3B cells were treated with $10 \mu \mathrm{M} 5$-Aza-dC, $0.25 \mathrm{~g} / \mathrm{ml} \mathrm{TSA}$ and $10 \mu \mathrm{M}$ DZNep. Expression of FLH1 was evaluated by reverse transcription quantitative polymerase chain reaction. Each column is presented as the mean \pm SD from 3 independent experiments. (B) HepG2 cells were treated with 5-Aza-dC, TSA and DZNep. Data was presented as mean \pm SD from 3 independent experiments. ${ }^{*} \mathrm{P}<0.05,{ }^{* *} \mathrm{P}<0.01 .5$-Aza-dC, 5-Aza-2' deoxycytidine; SD, standard deviation; TSA, Trichostatin A; DZNep, 3-deanzaneplanocin A.

$\mathbf{A}$
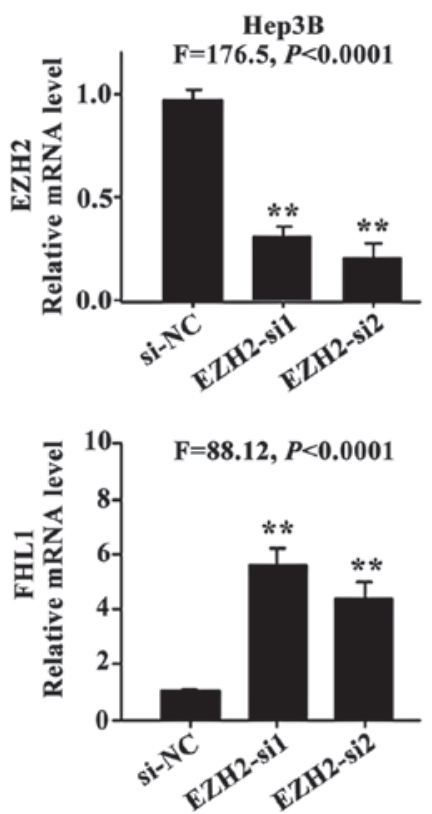

C

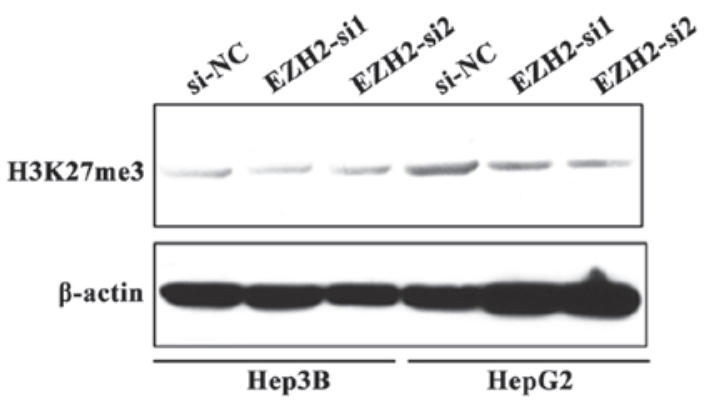

B
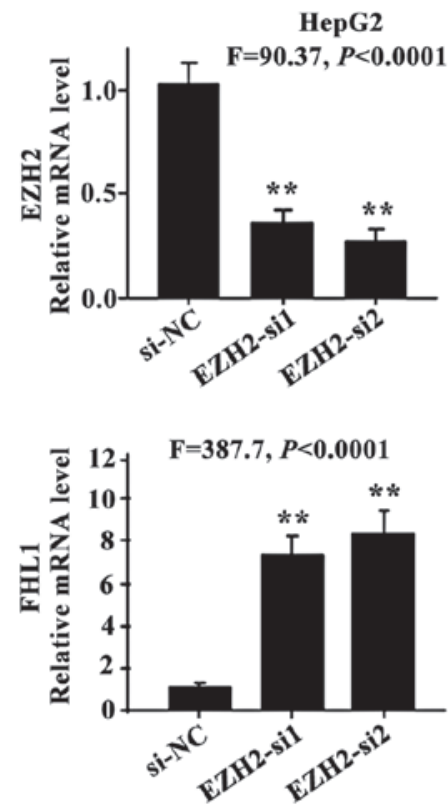

D

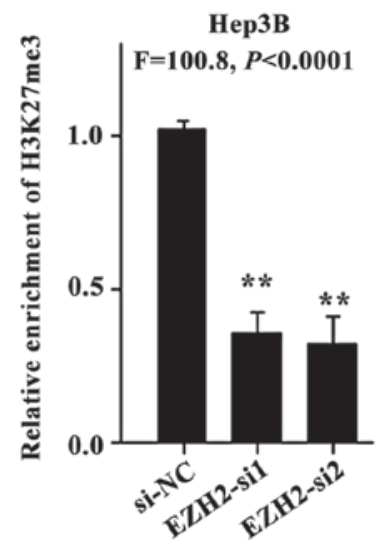

HepG2

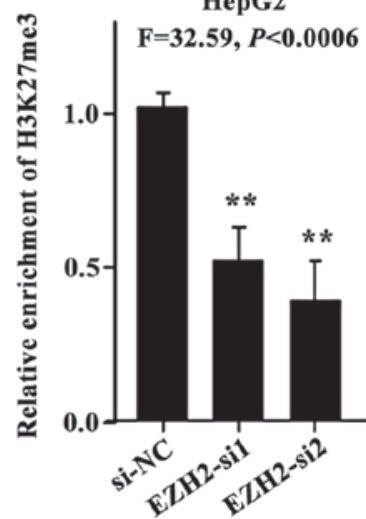

Figure 3. EZH2-mediated H3K27me3 was involved in the repression of FHL1 in HCC cell lines. (A and B) A total of 2 siRNAs against EZH2 were employed to silence EZH2 expression (upper), and FHL1 expression level was measured (bottom) in Hep3B and HepG2 cells. (C) Expression of H3K27me3 was detected by western blotting in Hep3B and HepG2 cells. (D) For the ChIP assay, quantitative polymerase chain reaction was used to analyze H3K27me3 enrichment on FHL promoter upon EZH2 knockdown in Hep3B and HepG2 cells. Fold of enrichment of the ChIP assay was calculated with reference to siNC. Data are presented as the mean \pm standard deviation. All comparisons were performed relative to si-NC control, and statistical significance was determined by two-tailed Student's t-test of two groups of data. FHL, four and a half LIM domains; ChIP, chromatin immunoprecipitation; EZH2, enhancer of zeste homolog 2; siNC, small interfering RNA negative control. 


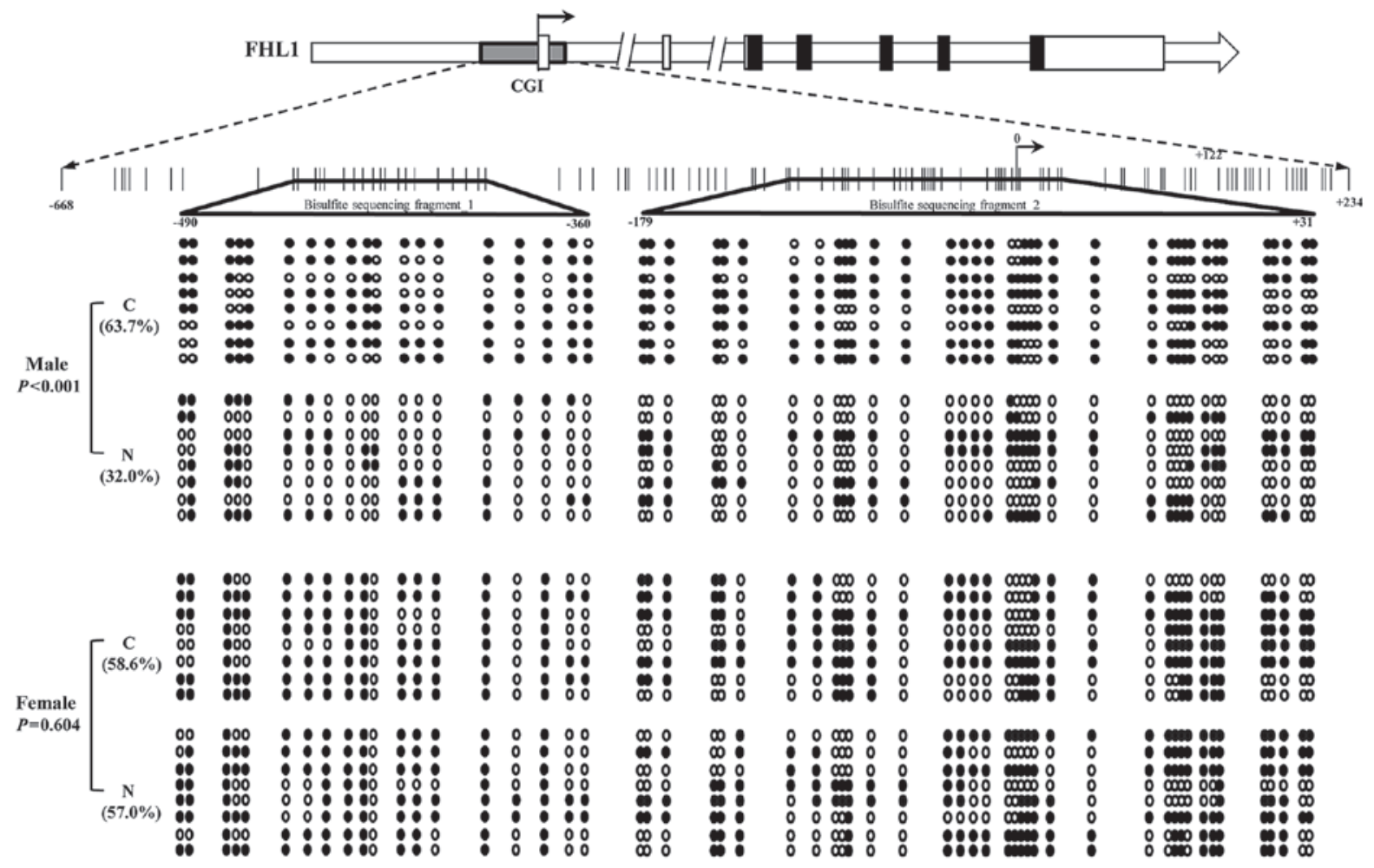

Figure 4. FHL1 promoter methylation analysis in liver cancer specimens based on bisulfite sequencing. A total of $19 \mathrm{CpG}$ dinucleotides located in the promoter region from -490 to -360 and $35 \mathrm{CpG}$ dinucleotides located in the promoter region from -179 to +31 , are represented by circles. Black and white represent the methylated and unmethylated CpG dinucleotides, respectively. FHL1, four and a half LIM domains 1.

A

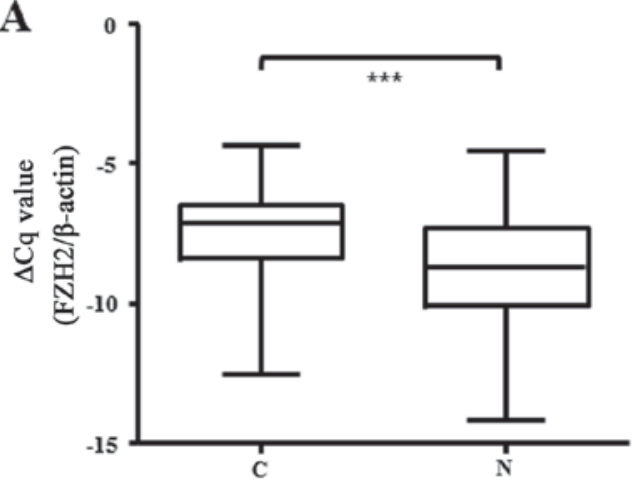

C

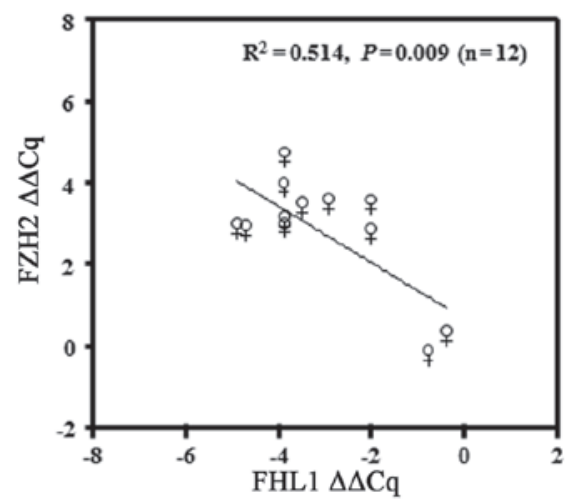

B

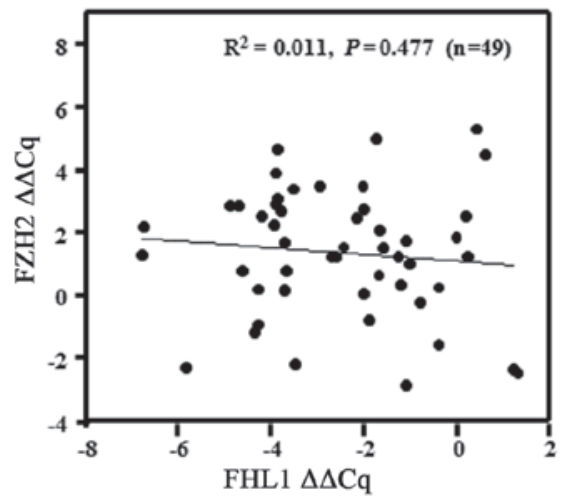

D

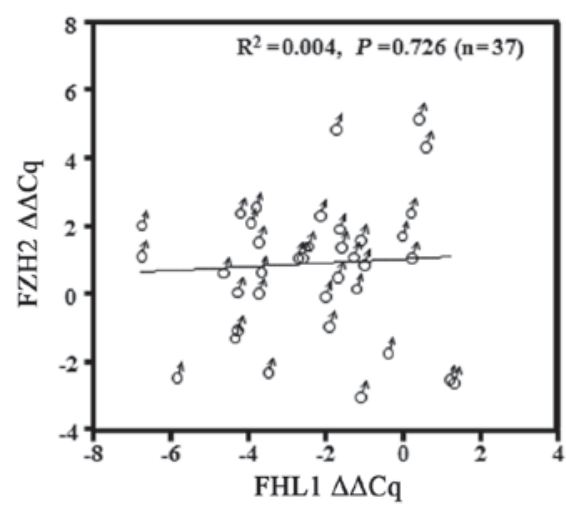

Figure 5. The correlation between EZH2 and FHL1 expression in human liver cancer. (A) Forty-nine liver cancer samples were examined for EZH2 mRNA expression by qPCR. P-value was calculated by two-tailed Student's t-test. (B-D) Correlations between FHL1 and EZH2 expression were calculated in (B) 49 paired samples of patients with liver cancer, categorized into $(C)$ female $(n=12)$ and $(D)$ male $(n=47)$ patients. Expression level of EZH2 and FHL1 in paired liver cancer samples was represented by $\Delta \Delta$ Cq. Linear regression analysis was performed using GraphPad Prism5. ${ }^{* * *} \mathrm{P}<0.001$. HCC, hepatocellular carcinoma; FHL1, four and a half LIM domains 1; N, adjacent non-cancerous livers; C, HCC specimens; EZH2, enhancer of zeste homolog 2. 
A
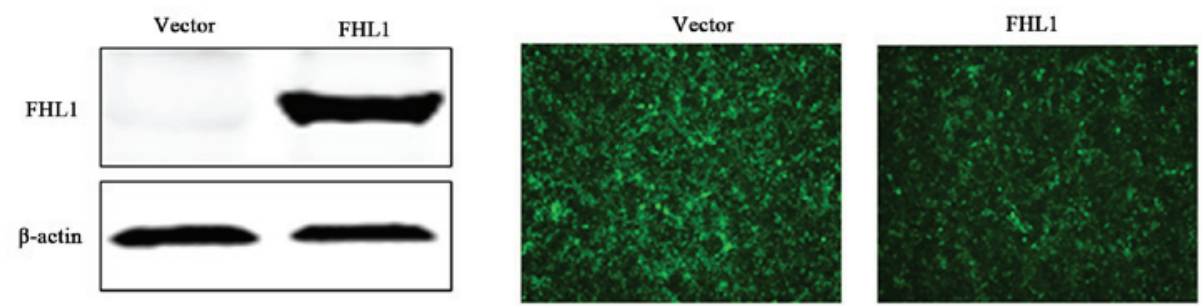

B
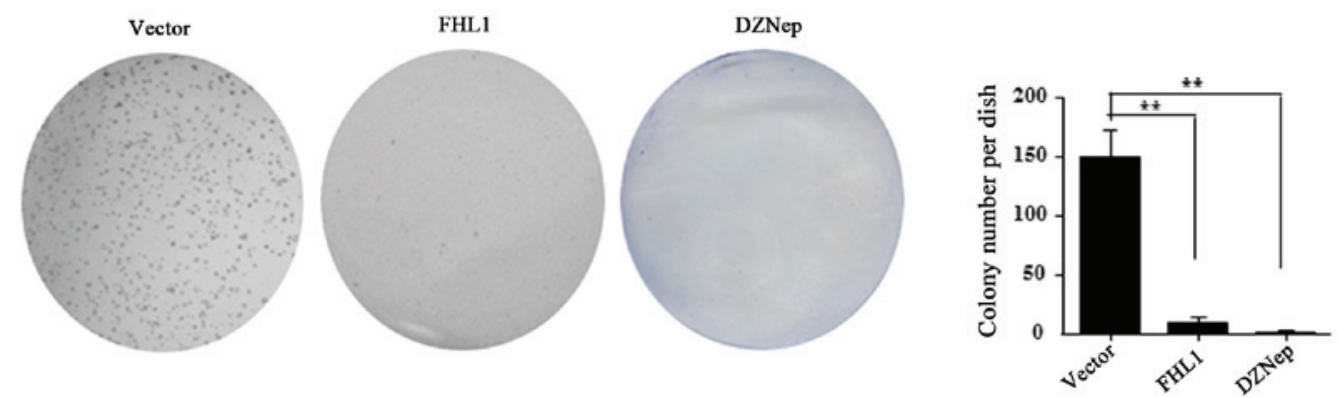

C
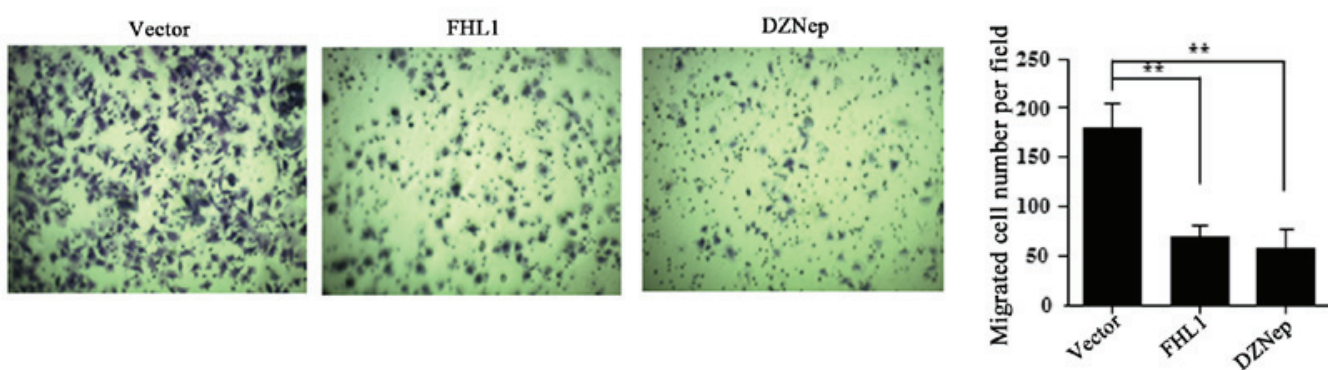

Figure 6. The suppressive effects of overexpressed FHL1 on hepatocellular carcinoma cell proliferation and migration of Hep3B. (A) Expression and infection efficiency of an adenovirus expression vector of FHL1 were evaluated by western blot assay and green fluorescent protein observation in Hep3B cells. (B) Anchorage-dependent colony formation assay was performed upon FHL1 overexpression and DZNep treatment respectively. P-values were calculated by a two-tailed t-test. (C) Cell migration was evaluated with a Transwell assay upon FHL1 overexpression and DZNep treatment, respectively. The permeable cells were stained with crystal violet and counted, and the results were statistically analyzed with a two-tailed t-test. ${ }^{* *} \mathrm{P}<0.01$. DZNep, 3-deanzaneplanocin A; FHL1, four and a half LIM domains 1.

FHL1 and EZH2, the present study examined EZH2 mRNA expression in 49 paired tissue samples. The results showed that EZH2 was significantly upregulated in the aforementioned cohort of samples with FHL1 downregulation (Fig. 5A). The present study then additionally analyzed the association between FHL1 downregulation and EZH2 upregulation, and failed to identify a significant association between the expression of the 2 genes $(\mathrm{P}=0.477$; Fig. 5B). Notably, an inverse correlation between FHL1 and EZH2 was observed in the samples of the female patients with $\mathrm{HCC}(\mathrm{P}=0.009$; Fig. $5 \mathrm{C})$, while this was not observed the samples from male patients $(\mathrm{P}=0.726$; Fig. 5D), suggesting the role of EZH2 upregulation in suppressing FHL1 expression in female patients with liver cancer.

FHL1 inhibits cell proliferation and migration in vitro. The present study then investigated the effect of FHL1 overexpression on HCC cell growth. For FHL1 overexpression, the recombinant adenovirus Ad-FHL1 tagged with GFP was transduced into Hep3B cells. After 3 days, FHL1 was overexpressed and almost $100 \%$ transduction efficiency was observed, as indicated by western blotting assay and GFP protein observation in Hep3B cells (Fig. 6A). To reveal the role of FHL1, the present study also performed anchorage-dependent colony formation and migration assays. The results demonstrated that FHL1 overexpression and DZNep treatment significantly inhibited the level of cell colony formation of Hep3B compared to the Ad-GFP empty vector control (Fig. 6B). Furthermore, the cell migration assay indicated that FHL1 overexpression or DZNep treatment significantly suppressed cell migration ability compared with the control cells (Fig. 6C). These findings suggest that EZH2 is involved in suppressing FHL1.

\section{Discussion}

FHL1 has a tumor suppressive role in a number of types of human cancer $(7-9,12)$. The findings of the present study reveal that EZH2 may act as a regulator of FHL1 expression in human liver cancer. FHL1 was identified as the first member of the fragile tumor suppressor gene on chromosome $\mathrm{X}$, and is inactivated by DNA methylation (23). Downregulation of FHL1 in tumor samples has been reported in breast (24), gastric (8) and lung cancers (7). A previous study revealed that EZH2 is a catalytic subunit of the epigenetic regulator PRC2, which trimethylates Lys27 of histone $\mathrm{H} 3$, leading to the silencing of target genes that are involved in numerous biological processes, including tumor progression (16). Overexpression of EZH2 has been detected in a range of types of cancer, and is associated 
with tumor malignancy $(14,15)$ via the epigenetic silencing of tumor and metastasis suppressor genes $(25,26)$. However, FHL1 downregulation was not significantly associated with clinicopathological characteristics of patients applied in the present study. With respect to the role of FHL1 in tumor initiation and progression, numerous studies concluded that FHL1 inhibits the growth of cancer cells, transforms fibroblasts and suppress the migration and invasion of bladder cancer cells $(7,12,13,27)$. The data obtained in the present study is in line with previous reports, and demonstrates that FHL1 overexpression inhibits cell proliferation and migration in HCC cells.

Primary liver cancer comprises HCC, intrahepatic cholangiocarcinoma and other rare tumors, notably fibrolamellar carcinoma and hepatoblastoma (28). At present, few studies have reported the expression level of FHL1 in ICC, fibrolamellar carcinoma and hepatoblastoma. The present study was also limited to HCC, due to rare incidence of other pathological types. However, the epigenetic regulations of FHL1 were investigated in the hepatoblastoma HepG2 cell line, which indicated that FHL1 was dysregulated by similar epigenetic mechanisms. In conclusion, the present study showed that DNA methylation and EZH2-induced H3K27me3 is associated with the epigenetic repression of the FHL1 tumor suppressor gene in HCC.

\section{Acknowledgements}

The present study was supported by Applied Basic Research Programs of Science and Technology Department of Suzhou (grant no. SYS201447) and Youth Science and Technology Project of Suzhou (grant no. kjxw2014001).

\section{References}

1. Ferlay J, Soerjomataram I, Dikshit R, Eser S, Mathers C, Rebelo M, Parkin DM, Forman D and Bray F: Cancer incidence and mortality worldwide: Sources, methods and major patterns in GLOBOCAN 2012. Int J Cancer 136: E359-E386, 2015.

2. Scaggiante B, Kazemi M, Pozzato G, Dapas B, Farra R, Grassi M, Zanconati $\mathrm{F}$ and Grassi G: Novel hepatocellular carcinoma molecules with prognostic and therapeutic potentials. World J Gastroenterol 20: 1268-1288, 2014.

3. Hassler MR and Egger G: Epigenomics of cancer-emerging new concepts. Biochimie 94: 2219-2230, 2012.

4. Sandoval J and Esteller M: Cancer epigenomics: Beyond genomics. Curr Opin Genet Dev 22: 50-55, 2012.

5. Ng EK, Lee SM, Li HY, Ngai SM, Tsui SK, Waye MM, Lee CY and Fung KP: Characterization of tissue-specific LIM domain protein (FHL1C) which is an alternatively spliced isoform of a human LIM-only protein (FHL1). J Cell Biochem 82: 1-10, 2001

6. Morgan MJ and Madgwick AJ: The LIM proteins FHL1 and FHL3 are expressed differently in skeletal muscle. Biochem Biophys Res Commun 255: 245-250, 1999.

7. Niu C, Liang C, Guo J, Cheng L, Zhang H, Qin X, Zhang Q, Ding L, Yuan B, Xu X, et al: Downregulation and growth inhibitory role of FHL1 in lung cancer. Int J Cancer 130: 2549-2556, 2012.

8. Xu Y, Liu Z and Guo K: Expression of FHL1 in gastric cancer tissue and its correlation with the invasion and metastasis of gastric cancer. Mol Cell Biochem 363: 93-99, 2012.
9. Sakashita K, Mimori K, Tanaka F, Kamohara Y, Inoue H, Sawada T, Hirakawa K and Mori M: Clinical significance of loss of Fhl1 expression in human gastric cancer. Ann Surg Oncol 15: 2293-2300, 2008.

10. Li X, Jia Z, Shen Y, Ichikawa H, Jarvik J, Nagele RG and Goldberg GS: Coordinate suppression of Sdpr and Fhl1 expression in tumors of the breast, kidney, and prostate. Cancer Sci 99: 1326-1333, 2008.

11. Spatz A, Borg C and Feunteun J: X-chromosome genetics and human cancer. Nat Rev Cancer 4: 617-629, 2004.

12. Ding L, Wang Z, Yan J, Yang X, Liu A, Qiu W, Zhu J, Han J, Zhang H, Lin J, et al: Human four-and-a-half LIM family members suppress tumor cell growth through a TGF-beta-like signaling pathway. J Clin Invest 119: 349-361, 2009.

13. Shen Y, Jia Z, Nagele RG, Ichikawa H and Goldberg GS: SRC uses Cas to suppress Fhl1 in order to promote nonanchored growth and migration of tumor cells. Cancer Res 66: 1543-1552, 2006.

14. Geng J, Li X, Zhou Z, Wu CL, Dai M and Bai X: EZH2 promotes tumor progression via regulating VEGF-A/AKT signaling in non-small cell lung cancer. Cancer Lett 359: 275-287, 2015.

15. Zingg D, Debbache J, Schaefer SM, Tuncer E, Frommel SC, Cheng P, Arenas-Ramirez N, Haeusel J, Zhang Y, Bonalli M, et al: The epigenetic modifier EZH2 controls melanoma growth and metastasis through silencing of distinct tumour suppressors. Nat Commun 6: 6051, 2015.

16. Li LY: EZH2: Novel therapeutic target for human cancer. Biomedicine (Taipei) 4: 1, 2014.

17. Cao R, Wang L, Wang H, Xia L, Erdjument-Bromage H, Tempst P, Jones RS and Zhang Y: Role of histone H3 lysine 27 methylation in Polycomb-group silencing. Science 298: 1039-1043, 2002.

18. Xie CR, Li Z, Sun HG, Wang FQ, Sun Y, Zhao WX, Zhang S, Zhao WX, Wang XM and Yin ZY: Mutual regulation between $\mathrm{CHD} 5$ and $\mathrm{EZH} 2$ in hepatocellular carcinoma. Oncotarget 6: 40940-40952, 2015.

19. Sasaki M, Yamaguchi J, Itatsu K, Ikeda H and Nakanuma Y: Over-expression of polycomb group protein EZH2 relates to decreased expression of p16 INK4a in cholangiocarcinogenesis in hepatolithiasis. J Pathol 215: 175-183, 2008.

20. López-Terrada D, Cheung SW, Finegold MJ and Knowles BB: Hep G2 is a hepatoblastoma-derived cell line. Hum Pathol 40: $1512-1515,2009$.

21. Livak KJ and Schmittgen TD: Analysis of relative gene expression data using real-time quantitative PCR and the 2(-Delta Delta C(T)) method. Methods 25: 402-408, 2001.

22. Paradis V: Histopathology of hepatocellular carcinoma. Recent Results Cancer Res 190: 21-32, 2013.

23. Asada K, Ando T, Niwa T, Nanjo S, Watanabe N, Okochi-Takada E, Yoshida T, Miyamoto K, Enomoto S, Ichinose M, et al: FHL1 on chromosome $\mathrm{X}$ is a single-hit gastrointestinal tumor-suppressor gene and contributes to the formation of an epigenetic field defect. Oncogene 32: 2140-2149, 2013.

24. Ding L, Niu C, Zheng Y, Xiong Z, Liu Y, Lin J, Sun H, Huang K, Yang W, Li X and Ye Q: FHL1 interacts with oestrogen receptors and regulates breast cancer cell growth. J Cell Mol Med 15: 72-85, 2011.

25. Wang C, Liu X, Chen Z, Huang H, Jin Y, Kolokythas A, Wang A, Dai Y, Wong DT and Zhou X: Polycomb group protein EZH2-mediated E-cadherin repression promotes metastasis of oral tongue squamous cell carcinoma. Mol Carcinog 52: 229-236, 2013.

26. Cao Q, Yu J, Dhanasekaran SM, Kim JH, Mani RS, Tomlins SA, Mehra R, Laxman B, Cao X, Yu J, et al: Repression of E-cadherin by the polycomb group protein EZH2 in cancer. Oncogene 27: 7274-7284, 2008 .

27. Matsumoto M, Kawakami K, Enokida H, Toki K, Matsuda R, Chiyomaru T, Nishiyama K, Kawahara K, Seki N and Nakagawa M: CpG hypermethylation of human four-and-a-half LIM domains 1 contributes to migration and invasion activity of human bladder cancer. Int J Mol Med 26: 241-247, 2010.

28. Sia D, Villanueva A, Friedman SL and Llovet JM: Liver cancer cell of origin, molecular class, and effects on patient prognosis. Gastroenterology 152: 745-761, 2017. 